\title{
Análise tridimensional de esforços em elementos rompedores de semeadoras de plantio direto
}

\author{
Tridimentional efforts analyses of furrow opening in no tillage seeder \\ Renildo Luiz Mion ${ }^{\text {I* }}$ Sérgio Hugo Benez ${ }^{\text {II }}$ Carlos Alberto Viliotti ${ }^{\text {I }}$ \\ João Batista Moreira' ${ }^{I}$ Nilson Salvador ${ }^{\text {III }}$
}

\section{RESUMO}

As informações da avaliação de mecanismos de abertura de sulco de semeadoras de plantio direto auxiliam as empresas no dimensionamento de ferramentas rompedoras do solo, que buscam menor requerimento energético. Este trabalho foi realizado num solo classificado como Latossolo Vermelho com o objetivo de avaliar tridimensionalmente os esforços dos mecanismos de abertura de sulco de semeadoras adubadoras de plantio direto. $O$ delineamento experimental utilizado foi o de blocos ao acaso com cinco tratamentos, sendo cinco mecanismos de abertura de sulco (disco liso - DL, disco corrugado - DC, disco ondulado - DO, disco duplo DD e haste sulcadora - H). Para mensurar os esforços dos mecanismos de abertura de sulco, foi utilizado um dinamômetro com sensibilidade nas direções horizontal, transversal e vertical e no momento fletor e torsor. A avaliação mostrou que a interação entre mecanismos de abertura de sulco e carga vertical não foi significativa, entretanto os fatores foram significativos quando analisados individualmente. $O$ resultado individual dos mecanismos de abertura de sulco mostrou que o disco ondulado proporcionou maior área de mobilização do solo, a haste sulcadora apresentou o menor valor de força vertical e o disco duplo e a haste sulcadora obtiveram o menor esforço horizontal.

Palavras-chave: semeadora, momento fletor, momento torsor, plantio direto, transdutor

\section{ABSTRACT}

Information from evaluation mechanisms of opening furrows seedlings in no-tillage systems helps companies todesign tools soil, seeking for lower energy requirement. This work was achieved in a Red Latosol soil in order to evaluate dimensional efforts of mechanisms opening furrows. The experimental design used was a complete randomized block with 5 projects and five mechanisms of furrow opening (even disk - DL, corrugated disk - DC, ondulated disk - DO, double disk - DD and furrow rod - H). To measure forces, a dynamometer with sensitivity in draught, transverse and vertical directions and torsor e fletor moment were used. The strength need to be consider in individual results of opening mechanisms furrows in tools designed for seeders The rod needs little effort to penetrate the vertical soil due to the suction. The ondulated disk provides greater mobilization of soil due to the project design of the tool.

Key words: seeders, moment fletor, moment torsor, no-tillage, transducer.

\section{INTRODUÇÃo}

O estudo detalhado dos esforços a que são submetidos os mecanismos sulcadores de semeadoras adubadoras é de grande importância para adequar e melhorar o desempenho nos diferentes tipos de solos e os resíduos provenientes de diversas culturas. Sob resíduos de milho e sorgo, o mecanismo sulcador do tipo disco de corte apresentou os maiores valores de força vertical devido à resistência que os resíduos oferecem. Entretanto, para as coberturas de triticale e nabiça, o maior valor encontrado foi para o disco ondulado, pois esse aumento está relacionado com a resistência superficial do solo e não da palha, de acordo com relato de SILVA (2007). Já os sulcadores do tipo

'Departamento Engenharia Agrícola, Centro de Ciências Agrárias (CCA), Universidade Federal do Ceará (UFC), Campus do Pici, Bloco 804, 60455-970, Fortaleza, CE, Brasil. E-mail: rmion@ufc.br.*Autor para correspondência.

IIDepartamento de Engenharia Rural, Faculdade de Ciências Agronômicas (FCA), Universidade Estadual Paulista (UNESP), Botucatu, SP, Brasil.

IIIDepartamento de Engenharia, Universidade Federal de Lavras (UFLA), Lavras, MG, Brasil. 
hastes têm maior demanda de força de tração, de acordo com TESSIER et al. (1989), porque trabalham a profundidades maiores que os discos.

De acordo com WELLS et al.(1980), podese determinar a direção e a magnitude da força resultante de um implemento qualquer desde que se conheça a magnitude das forças verticais e horizontais atuantes sobre este. Ao avaliar diferentes mecanismos sulcadores do tipo disco, SILVA (2007) observou que, para as coberturas de milho, sorgo e aveia preta, o disco de corte liso proporcionou os maiores valores de força transversal quando comparados ao disco ondulado e duplo, relatando que o disco liso possui menor área de contato com o solo, com isso, qualquer deslocamento transversal implica maior momento transversal.

Estudando esforços tridimensionais em uma semeadora adubadora acoplada aos três pontos do sistema hidráulico, CASÃO JR. et al. (2000) observaram que a força de tração apresentou uma magnitude média equivalente a $98 \%$ da força resultante, significando que, nos estudos de desempenho de máquinas em que somente esse parâmetro é determinado, a informação aproxima-se em muito da força resultante. Em relação à mobilização do solo de hastes sulcadoras, OLIVEIRA et al. (2000) verificaram que diferentes desenhos das hastes afetaram a força vertical e horizontal, seus valores específicos e a potência, demonstrando que é possível alterar esses parâmetros com mudanças nos projetos. O aumento no ângulo da haste proporcionou maior força vertical, e valores ao redor de $25^{\circ}$ a $30^{\circ}$ obtiveram menores requerimentos de força vertical, conforme CHAUDHURI (2001). O mesmo autor conclui que o ângulo de $130^{\circ}$ das hastes ofereceu maior estabilidade da profundidade em solo arenoso, enquanto os discos de corte são mais satisfatórios para o preparo convencional devido à menor necessidade de força e menor mobilização do solo. Avaliando as forças atuantes em hastes sulcadoras de semeadoras de plantio direto, SÁNCHEZ-GIRÓN et al. (2005) observaram que o ângulo da haste com $50^{\circ}$ proporcionou menores valores de força vertical e horizontal quando comparados aos ângulos de $112^{\circ}$, pois os ângulos menores facilitam a penetração no solo, porém mobilizam maior área.

Portanto, este trabalho teve como objetivo analisar individualmente os esforços tridimensionais em diferentes mecanismos de abertura de sulco utilizados por diferentes semeadoras adubadoras de plantio direto num solo de textura arenosa.

\section{MATERIAL E MÉTODOS}

O experimento foi instalado e conduzido em área da Fazenda Experimental São Manuel, pertencente à Faculdade de Ciências Agronômicas FCA/UNESP, Campus de Botucatu, São Paulo (SP), cuja localização geográfica está definida pelas coordenadas $22^{\circ} 46^{\text {' }}$ Latitude Sul e 48 ${ }^{\circ} 5^{\prime}$ 'de Longitude Oeste, com altitude média de $740 \mathrm{~m}$.

A área estava sendo manejada no sistema de plantio direto há dois anos e sua vegetação foi dessecada, predominando Brachiaria (Brachiaria decumbens $L$.), com $8.028,60 \mathrm{~kg} \mathrm{ha}^{-1}$ da massa seca da cobertura vegetal. O solo da área foi classificado como Latossolo Vermelho (EMBRAPA, 1999) com relevo plano a ondulado e declividade média de $7 \%$.

Os resultados da análise granulométrica na camada de $0-150 \mathrm{~mm}$ apresentaram os valores médios de 850 , 90 e $60 \mathrm{~g} \mathrm{~kg}^{-1}$ para areia, silte e argila, respectivamente, sendo classificado como arenoso. A densidade do solo na camada de 0 - 0,15m apresentou valores médios de $1,42 \mathrm{~g} \mathrm{~kg}^{-1}$. O teor de água do solo no momento do teste de avaliação dos sulcadores foi de $0,26 \mathrm{~kg} \mathrm{~kg}^{-1}$, e a resistência mecânica à penetração na camada de $0-0,15 \mathrm{~m}$ foi de $1730 \mathrm{kPa}$.

Foi utilizado trator da marca John Deere, modelo $6600 \mathrm{~T}$, com seis cilindros, potência $89(\mathrm{~kW})$ a 2300rpm, tração dianteira auxiliar (TDA), com a barra porta ferramenta acoplada aos três pontos do sistema hidráulico, devidamente regulada e instrumentada com célula de carga e medidor de profundidade, desenvolvida por MION (2002). A velocidade média para determinação dos testes foi de $5,2 \mathrm{~km}^{-1}$.

Os mecanismos de abertura de sulco utilizados para a realização do ensaio estão disponíveis no mercado para comercialização, sendo os seguintes: disco de corte liso (DL) com 381mm de diâmetro, 3mm de espessura e ângulo de fio de $10,5^{\circ}$; disco duplo (DD) com dois discos planos de 381mm de diâmetro, $3 \mathrm{~mm}$ de espessura, posicionados um em relação ao outro a um ângulo de $10,5^{\circ}$, com eixo desencontrado; disco corrugado (DC) de $355 \mathrm{~mm}$ de diâmetro, $3 \mathrm{~mm}$ de espessura e ângulo de fio de $5^{\circ}$; disco ondulado com 402mm de diâmetro, $3 \mathrm{~mm}$ de espessura e ângulo de fio de $12^{\circ}$; e haste sulcadora $(\mathrm{H})$ com espessura de $9 \mathrm{~mm}$, largura da ponteira de $28 \mathrm{~mm}$, ângulo vertical da ponteira $18,5^{\circ}$, com ângulo horizontal de $16^{\circ}$.

Para determinar a profundidade de trabalho dos mecanismos de abertura de sulco, utilizou-se um sistema composto por uma cremalheira acoplada a um potenciômetro de cinco volts e 10 voltas (para medir o deslocamento vertical da haste). Os sinais gerados pelos sensores foram armazenados e monitorados por 
um sistema de aquisição de dados fabricado pela Campbell Scientific, Inc., modelo “CR 21 X micrologger”, com uma freqüência de $10 \mathrm{~Hz}$.

O experimento foi constituído por cinco tratamentos em blocos ao acaso com quatro repetições, totalizando 20 parcelas experimentais. A parcela possuía $15 \mathrm{~m}$ de comprimento e $3 \mathrm{~m}$ de largura, perfazendo um total de $45 \mathrm{~m}^{2}$. Entre os blocos foi deixado um carreador com $10 \mathrm{~m}$ para manobras e estabilização do equipamento antes do início da aquisição de dados. O sistema de aquisição de dados "micrologger $21 \mathrm{X}$ ” foi usado nos experimentos para monitorar e gravar continuamente os sinais gerados pelos sensores, a uma freqüência previamente definida.

A área transversal de solo mobilizado $\left(\mathrm{cm}^{2}\right)$ foi obtida por meio de um perfilômetro de varetas, com espaçamento entre varetas de $0,01 \mathrm{~m}$, que determina o perfil mobilizado do solo. Para os cálculos, foi utilizada uma mesa digitalizadora, marca Digicom.

As variáveis estudadas foram: Força vertical (Fy), força horizontal (Fx), força transversal (Fl), Forças resultantes (XY, XZ e YZ) e momentos (My - Torção; $\mathrm{MZ}$ - Flexão). Os resultados obtidos foram tabulados e submetidos à análise de variância, pelo teste $\mathrm{F}$ no nível de 5\% de probabilidade e pelo teste Tukey para comparação das médias com o nível de probabilidade de 5\%. O pacote estatístico utilizado foi o SAEG.

Para determinar a necessidade de forças e os momentos solicitados pelos mecanismos sulcadores, foi utilizado um dinamômetro conforme figura 1, com sensibilidade variável para cada força solicitada e escala nominal de 25000 N. A determinação das variáveis foi calculada por meio da média aritmética de todos os valores obtidos e armazenados no sistema de aquisição de dados de cada parcela pelo número de dados coletados de acordo com a freqüência utilizada nos sensores, conforme Eq. (1):

$F=\sum_{i=1}^{n} \frac{F i}{n} \times 10$

em que:

$\mathrm{F}=$ força média $(\mathrm{N})$

$\mathrm{Fi}$ = força instantânea (kgf)

$\mathrm{n}=$ número de dados registrados

$10=$ constante

\section{RESULTADOS E DISCUSSÃO}

Forças horizontal, vertical e transversal

O mecanismo de abertura de sulco do tipo disco ondulado obteve a maior necessidade de força horizontal para vencer a resistência do solo (Tabela 1), possivelmente pelo desenho do disco e por sua ação dinâmica no solo ser diferente dos demais e, de certo modo, mobilizando maior área de solo. No entanto, na obtenção da força vertical, o seu desempenho foi semelhante ao mecanismo de abertura de sulco do tipo haste e disco liso. Os resultados encontrados para o

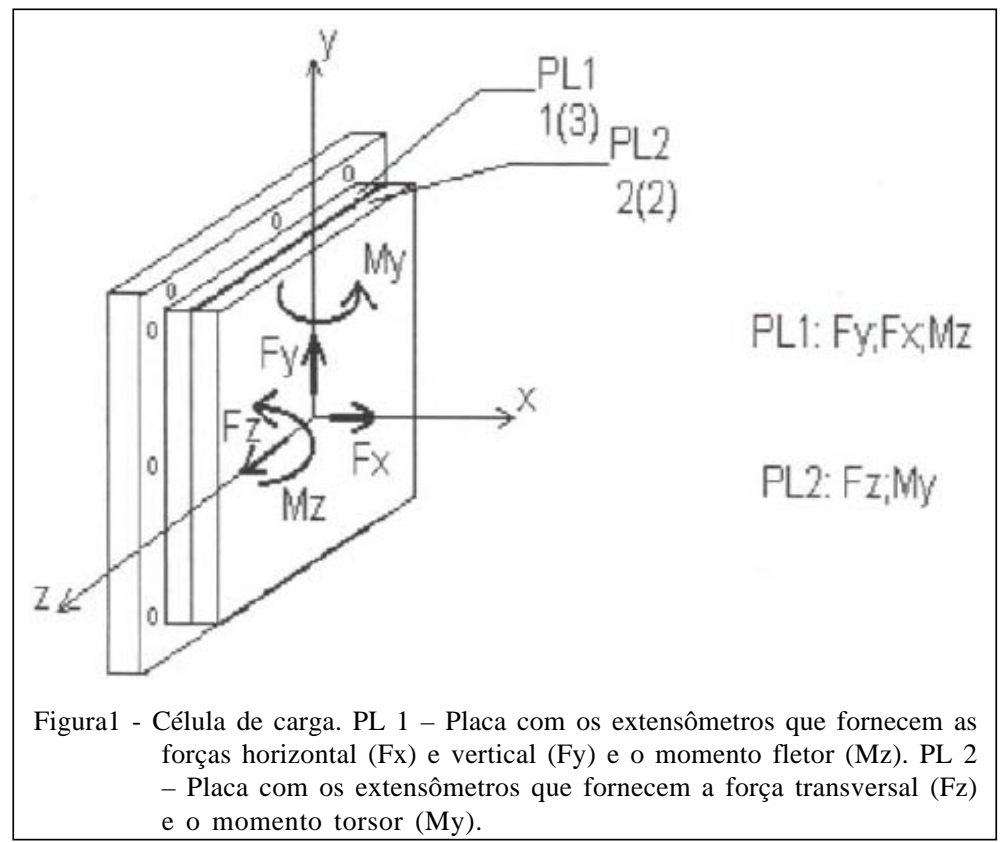

Ciência Rural, v.39, n.5, ago, 2009. 
Tabela 1 - Valores médios de força horizontal $(\mathrm{N})$, vertical $(\mathrm{N})$ e transversal(N) encontrados para os diferentes mecanismos sulcadores.

\begin{tabular}{lccc}
\hline Mecanismos de abertura de sulco & Força Horizontal (N) (Fx) & Força Vertical (N) (Fy) & Força Transversal (N) (Fz) \\
\hline Disco Ondulado & $861,20 \mathrm{a}$ & $-192,40 \mathrm{bc}$ & $-469,70 \mathrm{~d}$ \\
Disco Liso & $466,50 \mathrm{~b}$ & $-236,60 \mathrm{ab}$ & $-8,20 \mathrm{c}$ \\
Disco Corrugado & $394,30 \mathrm{~b}$ & $-274,40 \mathrm{a}$ & $-29,00 \mathrm{c}$ \\
Haste sulcadora & $261,30 \mathrm{c}$ & $-189,50 \mathrm{c}$ & $442,00 \mathrm{a}$ \\
Disco Duplo & $188,80 \mathrm{c}$ & $-248,20 \mathrm{a}$ & $227,00 \mathrm{~b}$ \\
Média & 434,40 & $-228,20$ & 32,40 \\
\hline
\end{tabular}

Médias seguidas de mesmas letras minúsculas nas colunas não diferem entre si pelo Teste de Tukey em nível de 5\% de probabilidade.

disco liso estão abaixo dos encontrados por BIANCHINI \& MAGALHÃES (2008), quando avaliaram força horizontal e vertical de disco de corte com diâmetro de $610 \mathrm{~mm}$ e profundidade de trabalho de $80 \mathrm{~mm}$. O valor para força horizontal foi de $940 \mathrm{~N}$ e para força vertical foi de $3.540 \mathrm{~N}$.

A força horizontal em módulo para os mecanismos de abertura de sulco do tipo haste e o disco ondulado apresentaram os maiores valores de força transversal, resultado que pode ser explicado pela declividade do terreno (7\%), pelos movimentos laterais da direção do trator e pela carga vertical sobre os mecanismos sulcadores. Em algumas situações podem ocorrer depressões no terreno, assim as acelerações podem atingir duas vezes a aceleração da gravidade, conforme salienta CASÃO JR. (2000), estudando semeadoras acopladas aos três pontos do sistema hidráulico. Em relação ao disco duplo, o que pode ocorrer com a força transversal está relacionado ao desenho da ferramenta, pois qualquer depressão do solo em um dos discos pode influenciar a força transversal e qualquer movimento na direção faz com a força gire em cima do mecanismo sulcador.

A força transversal dos mecanismos de abertura de sulco do tipo disco corrugado e liso apresentou comportamento semelhante (Tabela 1). Os demais mecanismos tiveram uma tendência de deslocamento transversal em relação ao centro de resistência do trator. No caso do comportamento do mecanismo de abertura de sulco do tipo haste, está de acordo com o encontrado por HARRISON (1982), estudando hastes inclinadas e superiores para a força horizontal e vertical para a mesma profundidade estudada por SÁNCHEZ-GIRÓN et al. (2005).

Entretanto, pode-se observar que, para os mecanismos de abertura de sulco do tipo disco corrugado, liso e duplo, foi necessário empregar uma força vertical maior devido à resistência oferecida pelo solo à penetração e pela palha presente na superfície, concordando com resultados obtidos por PORTELLA (1983) e KUSHWAHA et al. (1986).

\section{Força resultante}

Observa-se que a força resultante em XY do mecanismo de abertura de sulco do tipo disco ondulado foi maior em relação aos demais em conseqüência do elevado valor da força horizontal (Tabela 2). Para os mecanismos de abertura de sulco do tipo liso e corrugado, o desempenho foi semelhante, e os mecanismos de abertura de sulco do tipo haste sulcadora e disco defasado obtiveram as menores forças resultantes, sendo esses valores semelhantes

Tabela 2 - Valores médios da força resultante (N) encontrados para os diferentes mecanismos sulcadores.

\begin{tabular}{lccc}
\hline Mecanismos de abertura de sulco & Resultante XY (N) & Resultante XZ (N) & Resultante YZ (N) \\
\hline Disco Ondulado & $882,43 \mathrm{a}$ & $983,50 \mathrm{a}$ & $507,50 \mathrm{a}$ \\
Disco Liso & $523,06 \mathrm{~b}$ & $466,57 \mathrm{~b}$ & $236,74 \mathrm{c}$ \\
Disco Corrugado & $480,38 \mathrm{~b}$ & $395,36 \mathrm{c}$ & $275,92 \mathrm{bc}$ \\
Haste sulcadora & $322,78 \mathrm{c}$ & $513,46 \mathrm{~b}$ & $480,90 \mathrm{a}$ \\
Disco Duplo & $311,84 \mathrm{c}$ & $295,25 \mathrm{c}$ & $336,25 \mathrm{~b}$ \\
Média & 504,19 & 530,32 & 367,40 \\
\hline
\end{tabular}

Médias seguidas de mesmas letras minúsculas nas colunas não diferem entre si pelo Teste de Tukey em nível de 5\% de probabilidade.

Ciência Rural, v.39, n.5, ago, 2009. 
aos encontrados por PORTELLA (1983). Além disso, os valores apresentaram magnitude média superior a $60 \%$ em relação à força horizontal. Isso mostra que o dimensionamento da haste sulcadora deverá levar em consideração a força resultante e não a força horizontal.

As forças resultantes em $\mathrm{XZ}$ seguem um comportamento semelhante aos comportamentos obtidos para as forças horizontais e laterais, destacando-se novamente um comportamento superior do disco ondulado em relação aos demais, mostrando a importância do dimensionamento de mecanismos sulcadores e levando-se em consideração as forças resultantes.

Pode-se observar que o desempenho dos mecanismos de abertura de sulco com relação à força resultante em YZ apresentou desempenho semelhante entre o disco ondulado e a haste, o que vem reforçar a dependência desse fator com relação à força transversal e vertical, influência da declividade, presença de palha e resistência do solo.

\section{Momento torsor e fletor}

O mecanismo de abertura de sulco do tipo disco ondulado obteve o maior valor em relação aos demais (Tabela 3). Isso se deve à força transversal, uma vez que o momento torsor representa a soma algébrica dos momentos gerados por cargas contidas ou que possuem componentes no plano YZ, perpendicular ao eixo $X$, o qual produz esforço que gira a seção em torno do eixo longitudinal, provocando tensões de cisalhamento.

O desempenho do disco ondulado provavelmente deve-se ao desenho do mecanismo de abertura de sulco, mostrando que deve ocorrer uma preocupação das empresas fabricantes com relação a esse esforço no momento de dimensionamento dos apoios dos sulcadores.

Os mecanismos de abertura de sulco do tipo disco liso e corrugado obtiveram desempenho semelhante conforme ocorrido na força transversal. $\mathrm{O}$ mecanismo tipo haste e o mecanismo disco duplo apresentaram os menores valores para o momento torsor, mostrando menor influência com relação às tensões de cisalhamento. Esse desempenho da haste pode ser explicado pela largura da sapata (ponteira), melhorando a estabilidade lateral, e o disco duplo possui dois de apoios.

Observa-se que não houve diferença estatística significativa entre os mecanismos de abertura de sulco (Tabela 3). É importante observar que a resistência oferecida pelo solo varia em função do tipo, do teor de água e da densidade e, nesse caso, do momento torsor e fletor. Assim, a declividade do terreno, a quantidade de palha e a carga vertical contribuem para o aumento dos esforços.

Área de seção transversal de solo mobilizado e profundidade dos mecanismos de abertura de sulco O disco ondulado teve uma maior área de solo mobilizada quando comparado aos demais mecanismos de abertura de sulco (Tabela 4), o que se deve ao desenho do disco e à maior área de contato com o solo. Para os demais mecanismos de abertura de sulco, não houve diferença estatística significativa. Os valores encontrados para a haste sulcadora estão próximos dos encontrados por SÁNCHEZ-GIRÓN et al. (2005) para a mesma profundidade e o mesmo tipo de solo. Quanto à profundidade operacional dos mecanismos, observou-se que a haste sulcadora atingiu maior profundidade devido à ação da ponteira que tem tendência de succionar a haste. O disco duplo atingiu menores profundidades devido às superfícies de contato da ferramenta com o solo ocorrer em dobro, concordando com KUSHWAHA \& FOSTER (1993).

\section{CONCLUSÃO}

No dimensionamento de ferramentas rompedoras para semeadoras, deve-se levar em consideração a força resultante. A haste sulcadora precisa de menor esforço vertical para penetrar no solo devido à sucção. O disco ondulado proporciona maior mobilização do solo.

Tabela 3 - Valores médios do momento ( $\mathrm{N} \mathrm{m}$ ) para os diferentes mecanismos sulcadores.

\begin{tabular}{lcc}
\hline Mecanismos de abertura de sulco & Momento torsor (N m) & Momento fletor (N m) \\
\hline Disco Ondulado & $1353,90 \mathrm{a}$ & $20,30 \mathrm{a}$ \\
Disco Liso & $729,50 \mathrm{~b}$ & $3,30 \mathrm{a}$ \\
Disco Corrugado & $720,80 \mathrm{~b}$ & $-16,90 \mathrm{a}$ \\
Haste sulcadora & $219,70 \mathrm{c}$ & $24,10 \mathrm{a}$ \\
Disco Duplo & $254,30 \mathrm{c}$ & 5,70 a \\
Média & 655,60 & 7,30 \\
\hline
\end{tabular}

Médias seguidas de mesmas letras minúsculas nas colunas não diferem entre si pelo Teste de Tukey em nível de 5\% de probabilidade.

Ciência Rural, v.39, n.5, ago, 2009. 
Tabela 4 - Valores médios de área de seção transversal de solo mobilizada $\left(\mathrm{cm}^{2}\right)$ e profundidade (cm).

\begin{tabular}{lcc}
\hline Mecanismos de abertura de sulco & Área de Seção Transversal de Solo Mobilizado $\left(\mathrm{cm}^{2}\right)$ & Profundidade $(\mathrm{cm})$ \\
\hline Disco Ondulado & $40,78 \mathrm{a}$ & $4,15 \mathrm{ab}$ \\
Disco Liso & $15,05 \mathrm{~b}$ & $3,45 \mathrm{bc}$ \\
Disco Corrugado & $14,14 \mathrm{~b}$ & $3,28 \mathrm{bc}$ \\
Haste sulcadora & $16,71 \mathrm{~b}$ & $4,68 \mathrm{a}$ \\
Disco Duplo & $12,21 \mathrm{~b}$ & $3,11 \mathrm{c}$ \\
Média & 19,77 & 3,73 \\
\hline
\end{tabular}

Médias seguidas de mesmas letras minúsculas nas colunas não diferem entre si pelo Teste de Tukey em nível de 5\% de probabilidade.

\section{REFERÊNCIAS}

BIANCHINI, A.; MAGALHÃES, P.S.G. Evaluation of coulters for cutting sugar cane residue in a soil bin. Biosystems Engineering, v.100, n.3, p.370-375, 2008. Disponível em: $<\mathrm{ht} \mathrm{t} \mathrm{p} \mathrm{:} \mathrm{/} \mathrm{/} \mathrm{w} \mathrm{w} \mathrm{w.} \mathrm{s} \mathrm{c} \mathrm{i} \mathrm{e} \mathrm{n} \mathrm{c} \mathrm{ed} \mathrm{i} \mathrm{r} \mathrm{e} \mathrm{c} \mathrm{t.} \mathrm{c} \mathrm{o} \mathrm{m/}$ science?_ob=ArticleURL\&_udi=B6WXV-4SRDFCP$1 \&$ _ us e r $=10 \&$ _ c over Dat e $=07 \% 2$ F $31 \% 2$ F 20 $08 \&$ \&doc $=9 \&$ \&mt $=$ high \&_orig $=$ browse \&_srch $=$ doc i n f o ( $\% \begin{array}{lllllllllllllllll}\% & 2 & \text { t } & \text { o c } & \% & 2 & 3 & 7 & 1 & 6 & 8 & \% & 2 & 3 & 2 & 0 & 0\end{array}$ \%23998999996\%23693147\%23FLA\%23display\%23Volume)\&_cdi= $7168 \&$ \& sort $=\mathrm{d} \&$ docanchor $=\&$ ct $=16 \&$ acct $=\mathrm{C} 0000502$ $21 \&$ \& v e r s i o n $=1 \&$ \& u r l V e r s i o $n=0 \&$. userid $=\overline{10} \& \mathrm{md} 5=$ f175ef4604361b0d5be1deaa177868e3>. Doi: 10.1016/j.biosystemseng.2008.04.012

CASÃO JUNIOR, R. et al. Análise tridimensional de esforços em semeadora de plantio direto. In: CONGRESSO BRASILEIRO DE ENGENHARIA AGRÍCOLA, 29., 2000, Fortaleza. Anais.. Fortaleza: Sociedade Brasileira de Engenharia Agrícola, 2000. CD ROM.

CASÃO JÚNIOR, R. Avaliação do desempenho da semeadora-adubadora Magnum 2850 PD no basalto paranaense. Londrina, Instituto Agronômico do Paraná, 1998 p.12-5. (circular n.105).

CHAUDHURI, D. Performance evaluation of various types of furrow openers on seed drills - a review. Journal of Agricultural Engineering Research, v.79, n.2, p.125-137, 2001. Disponível em: <http://www.sciencedirect.com/ science?_ob=ArticleURL\&_udi=B6WH1-45RFHNS S\&_user $=10 \&$ \& rdoc $=1 \&$ \& $\mathrm{mt}=\&$ \&_orig $=$ search \&_so $\mathrm{rt}=\mathrm{d} \& \mathrm{v}$ i e $\mathrm{w}=\mathrm{c} \&$ \& a c c t $=$ C $0000050221 \%$ \& v e r s i o n $=1 \&$ _ u r l V e r s i o $n=0 \&$ u s erid $=10 \& \mathrm{md} 5=90 \mathrm{f} 6 \mathrm{f} \overline{6} \mathrm{df} 26042$ eb $95062 \mathrm{~d} 2 \mathrm{cae} 615 \mathrm{f} 351>$. Doi:10.1006/jaer.2000.0688.

EMPRESA BRASILEIRA DE PESQUISA AGROPECUÁRIA. Centro Nacional de Pesquisas de Solos. Sistema Brasileiro de Classificação de Solos. Brasília, 1999. 412p.

HARRISON, H.P. Soil reactions from laboratory studies with na inclined blade. Transaction of the ASAE, v.28, p.7-33, 1982.

KUSHWARA, R.L., FOSTER, R.K. Field evaluation of grain drill furouw openers and press wheels for no till seeding. Canadian Journal of Agricultural Engineering, v.35, p.253-257, 1993.

KUSHWAHA, R.L. et al. Soil bin evaluation of disc coulters under no-till crop residue conditions. Transaction of the ASAE, v.29, n.1, p.40-44, 1986.
OLIVEIRA, M.F.B de. et al. Mobilização do solo por hastes sulcadoras de semeadoras-adubadoras de plantio direto. In: CONGRESSO BRASILEIRO DE ENGENHARIA AGRÍCOLA, 29. 2000, Fortaleza. Anais... Fortaleza: Sociedade Brasileira de Engenharia Agrícola, 2000. CD ROM.

MION, R.L. Desenvolvimento de equipamento para ensaio a campo de ferramentas de rompimento do solo testado com mecanismos de abertura de sulcos de semeadoras. 2002. 96f. Tese (Doutorado em Agronomia / Energia na Agricultura) - Curso de Pós-graduação em Agronomia, Faculdade de Ciências Agronômicas, Universidade Estadual Paulista.

PORTELLA, J.A. Um estudo preliminar de forças atuantes de elementos rompedores de semeadeiras comerciais. 1983. 69f. Dissertação (Mestrado em Engenharia Agrícola / Mecanização Agrícola) - Curso de Pós-graduação em Engenharia Agrícola, Universidade Estadual de Campinas.

PORTELLA, A.J.; FAGANELLO, A. Máquinas para semeadura direta: desempenho dos elementos rompedores de solo. Dirigente Rural, v.23, p.13-26, 1984.

SÁNCHEZ-GIRÓN, V. et al. Effect of soil compaction and water content on the resulting forces acting on three seed drill furrow openers. Soil and Tillage Research, v.81, n.1, p.2537, 2005. Disponível em : <http://www.sciencedirect.com/ science?_ob=ArticleURL\&_udi=B6TC6-4D2684T$3 \&$ u s e r $=10 \&$ c o v e r D a t e $=03 \% 2 \mathrm{~F} 3$ 1\%2F2005\&_rdoc $=4 \&$ \&mt $=$ high\&_orig=browse\&_srch=docinfo(\%23toc\%235162\%232005\%23999189998\%23578792\%2 3 F L A \% 23 d i s p l a y \% 23 V o l u m e ) \& c d i $=5162 \&$ s o r t $=$ d \& d o c a n c hor $=$ \&_ct $=12$ \&_acct $=\mathrm{C} 000050221 \&$ _version $=1$ \&_urlVersion $=0$ \&_userid=10\&md5=6f7c36a575bd676da956686489f52e73>. Doi:10.1016/j.still.2004.04.003.

SILVA, P.R.A. Semeadora adubadoras: mecanismos de corte de palha e cargas verticais aplicadas. 2007. 93f. Tese (Doutorado em Agronomia / Energia na Agricultura) Curso de Pós-graduação em Agronomia, Faculdade de Ciências Agronômicas, Universidade Estadual Paulista.

TESSIER, S. et al. Roughness meter to measure seed row geometry and soil disturbance. Transaction of the ASAE, v.32, p.1871-73, 1989

WELLS, L.G. et al. Ground driven powered tillage. Transaction of the ASAE, v.23, n.6, p.1383-1387, 1980. 